\title{
Do Disk Galaxies with Abnormally Low Mass-to-Light Ratios Exist?
}

\author{
(C) 2011 . A. S. Saburova ${ }^{1 *}$, D. V. Bizyaev ${ }^{1,2 * *}$, A. V. Zasov ${ }^{1 * * *}$ \\ Sternberg Astronomical Institute of Moscow State University, Universitetskii pr. 13, \\ Moscow, 119992 Russia $^{1}$ \\ Apache Point Observatory, New Mexico, $U S A^{2}$
}

\begin{abstract}
We performed the photometric $\mathrm{B}, \mathrm{V}$ and $\mathrm{R}$ observations of nine disk galaxies that were suspected in having abnormally low total mass-to-light (M/L) ratios for their observed color indices. We use our surface photometry data to analyze the possible reasons for the anomalous M/L. We infer that in most cases this is a result of errors in photometry or rotational velocity, however for some galaxies we cannot exclude the real peculiarities of the galactic stellar population. The comparison of the photometric and dynamical mass estimates in the disk shows that the low $\mathrm{M} / \mathrm{L}$ values for a given color of disks are probably real for a few our galaxies: NGC 4826 (Sab), NGC 5347 (Sab), and NGC 6814 $(\mathrm{Sb})$. The small number of such galaxies suggests that the stellar initial mass function is indeed universal, and that only a small fraction of galaxies may have a non-typical low-mass star depleted initial mass function. Such galaxies require more careful studies for understanding their star formation history.
\end{abstract}

Key words: galaxies, galactic disks, surface photometry, stellar initial mass function.

PACS codes: 98.52.Nr, 98.52.Sw, 98.62.Ai, 98.62.Lv, 98.62.Qz, 98.62.Hr

* E-mail:<saburovaann@gmail.com>

** E-mail: <dmbiz@apo.nmsu.edu>

*** E-mail: <a.v.zasov@gmail.com> 


\section{INTRODUCTION}

The mass-to-light $(M / L)$ ratio is a very important parameter that is determined by galactic stellar population (the distribution of stars in age, mass, and, to a lesser extent, stellar metallicity), dust (through affecting the galaxy's luminosity) and the relative mass of its nonstellar components. The latters include the dark matter, which is usually comparable in mass to the stellar component within the galaxy's optical boundaries (see, e.g., Khoperskov et al. 2010; Zasov et al. 2011; Bizyaev and Mitronova 2009), as well as the gas whose mass fraction can be significant in late-type galaxies. The stellar population models computed for certain accepted initial mass function (IMF) obviously predict $M / L_{B}$ and $M / L_{V}$ to be of the order of 1-10 solar units in the visible bands, and $M / L_{R}$ or $M / L_{K} \sim 0.5-2$ solar units in the near infrared, in dependence of the relative number of young stars (and therefore the galaxy's color) and the adopted IMF. The presence of the dark matter or the gas along with internal extinction can only increase the overal galactic $M / L$. Therefore, those galaxies whose total $M / L$ estimated within the optical boundaries looks lower than that provided by the stellar population modeling using the universal IMF (even ignoring any dark halo) are of especial interest. Such candidate galaxies were selected from the sample of objects with known rotational velocities by Saburova et al. (2009).

Since the reliable luminosity, color, and rotational velocity estimates are available not for all selected objects, the conclusion about the $M / L$ anomaly for each specific galaxy should be verified. An unusually low $M / L$ for a given color index, if confirmed, can point to the existence of stellar population with a non-standard IMF: such a galaxy must have a low relative number of stars with a mass less than the solar one because these stars determine the total mass of stellar population once have a little affect on the photometric features. This would imply the existence of special conditions for the formation of the the bulk of its stars. Note that the questions about the IMF universality and the possibility of a non-typical IMF shape are being actively discussed in the literature (see, e.g., Gilmore

2001; Kroupa 2002; Hoversten and Glazebrook 2008; Meureret al. 2009; Bastian et al. 2010; Dabringhausen et al. 2010).

In this paper we present the results of our photometry for nine galaxies selected from Saburova et al (2009) that were previously identified as presumably having low total $M / L$ ratios within the optical radius $R_{25}$ (the radius of the $25^{\mathrm{m}} / \square^{\prime \prime}$ isophote in $B$ ) and 
analyze some possible sources of errors in the observed quantities. We also compare the maximum rotation velocity of the disk component $v_{\max }$ disk, expected for a "normal" stellar population with the maximum observed rotation velocity $v_{\max }$. As a reference, we employ the model values of the mass-to-light ratios for the stellar population with Salpeter IMF following the evolutionary models by Bell \& de Jong (2001). The condition $v_{\max }$ disk $>v_{\max }$ suggests that the stellar population model with a regular IMFs is inapplicable and that the the real stellar disk is lighter than it is predicted by the stellar population models even if non-stellar components (dark matter and gas) are ignored. To verify the total luminosity estimate as well as the photometric parameters: color and inclination of the disk, we performed photometric observations of nine galaxies in which we suspect the abnormally low $M / L$.

\section{OBSERVATIONS AND DATA REDUCTION}

Photometric observations of the nine galaxies selected by Saburova et al. (2009) as galaxies with presumably low $M / L$ were conducted at the 0.5 -m Apache Point Observatory telescope in the $\mathrm{B}, \mathrm{V}$, and $\mathrm{R}$ bands. We observed the galaxies during photometric moonless nights in June, July, and October of 2009. Table 1 shows the observing log 11 The observing data were reduced in a standard way using MIDAS software package and were corrected for the bias and flat field. The telescope's wide field of view $\left(40^{\prime}\right)$ allowed the proper sky background subtraction using the starless areas in the field. The photometric standard stars from Landolt $(1992,2009)$ were observed during the same nights as the galaxies. The foreground stars were removed from the galactic images and replaced by the mean fluxes from the adjacent regions.

\section{RESULTS OF PHOTOMETRIC OBSERVATIONS}

Table 2 shows the derived total magnitudes and $(B-V)$ colors uncorrected for extinction. The total magnitudes were calculated from the fluxes in an aperture close to $R_{25}$ size. For the comparison, the table shows the apparent $B$ magnitudes and $(B-V)$ colors from Hyperleda database ${ }^{2}$ As follows from Table 2, our total magnitudes and those from Hyperleda agree well. NGC 4016 for which the NED databas $3^{3}$ provides apparently

\footnotetext{
${ }^{1}$ The listed distances correspond to the Hubble constant $H=75 \mathrm{kms}^{-1} \mathrm{Mpc}^{-1}$. For the nearby galaxy NGC 1569, we used the distance from Stil \& Israel (2002).

${ }^{2}$ http://leda.univ-lyon1.fr/.

${ }^{3}$ http://www.ned.ipac.caltech.edu/.
} 
Table 1: Log of observations.

\begin{tabular}{|c|c|c|c|c|c|}
\hline \multirow[t]{2}{*}{$\begin{array}{c}\text { Galaxy } \\
(1)\end{array}$} & \multirow[t]{2}{*}{$\begin{array}{c}\text { Date of observation } \\
(2)\end{array}$} & \multicolumn{3}{|c|}{$\begin{array}{c}\text { Band and exposure time, } \mathrm{s} \\
(3)\end{array}$} & \multirow[t]{2}{*}{$\begin{array}{c}\mathrm{D}, \mathrm{Mpc} \\
(4)\end{array}$} \\
\hline & & B & $\mathrm{V}$ & $\mathrm{R}$ & \\
\hline NGC1569 & 15.10 .09 & $3 \times 500$ & $3 \times 300$ & $3 \times 300$ & 2.2 \\
\hline NGC4016 & 14.06 .09 & 500 & 200 & 300 & 49.2 \\
\hline NGC4016 & 15.06 .09 & $2 \times 500$ & $2 \times 300$ & 2X300 & 49.2 \\
\hline NGC4214 & 15.06 .09 & $2 \times 500$ & $2 \times 300$ & $2 \times 300$ & 7 \\
\hline NGC4826 & 15.06 .09 & $2 \times 500$ & $2 \times 300$ & $2 \times 300$ & 7 \\
\hline NGC5347 & 15.06 .09 & 500 & 300 & 300 & 32.5 \\
\hline NGC5347 & 21.06 .09 & 500 & 300 & 300 & 32.5 \\
\hline NGC5921 & 15.06 .09 & 500 & 300 & 300 & 25.2 \\
\hline NGC5921 & 21.06 .09 & $2 \times 500$ & $2 \times 300$ & $2 \times 300$ & 25.2 \\
\hline NGC6814 & 21.06 .09 & $2 \times 500$ & $2 \times 300$ & $2 \times 300$ & 20.7 \\
\hline NGC7743 & 21.06 .09 & 500 & 300 & 300 & 24.7 \\
\hline UGC03685 & 15.10 .09 & $3 \times 500$ & $3 \times 300$ & $3 \times 300$ & 26.8 \\
\hline
\end{tabular}

erroneous value of $m_{B}$ corresponding to unrealistically low $(B-V)=0.03$ is an exception. We see a good agreement between the magnitudes and colors for our different nights (no more than $0.1^{m}$ and $0.04^{m}$ for the magnitudes and colors, respectively).

Table 2: Total magnitudes and colors.

\begin{tabular}{|c|c|c|c|c|c|}
\hline Galaxy & \multicolumn{2}{|c|}{$(B-V)_{\text {tot }}$} & \multicolumn{2}{c|}{$m_{B}$} & $m_{R}$ \\
\hline & this paper & Leda & this paper & Leda & \\
\hline NGC1569 & 0.83 & 0.83 & 11.8 & 11.8 & 10.4 \\
\hline NGC4016 & 0.32 & & 14.5 & 13.8 & 13.8 \\
\hline NGC4214 & 0.47 & 0.46 & 10.4 & 10.2 & 9.5 \\
\hline NGC4826 & 0.84 & 0.84 & 9.37 & 9.30 & 7.98 \\
\hline NGC5347 & 0.75 & 0.76 & 13.5 & 13.4 & 12.1 \\
\hline NGC5921 & 0.69 & 0.66 & 11.7 & 11.7 & 10.5 \\
\hline NGC6814 & 0.9 & 0.8 & 12.2 & 12.1 & 10.6 \\
\hline NGC7743 & 0.92 & 0.90 & 12.5 & 12.4 & 11 \\
\hline UGC03685 & 0.7 & & 12.8 & 13.1 & 11.6 \\
\hline
\end{tabular}

Along from the total magnitudes we obtained the radial profiles of isophotal flattening $(b / a)$ in the R-band images azimuthally averaged in elliptical rings (see Fig. 1). The radial surface brightness profiles in all three $B V R$-bands are plotted in Fig. 2 . The brightness profiles were corrected for extinction in the Galaxy using Schlegel et al. (1998), but they were not corrected for the disk orientation and internal extinction ${ }^{4}$ (the profiles

\footnotetext{
${ }^{4}$ Note that extinction inside the galaxies only slightly affects position of points on $M / L$-color diagram
} 
of NGC 1569 were not corrected for the extinction in the Galaxy due to the uncertainty in the correction, see below). The radial brightness and color profiles are shown in Figs. 2 and 3. The ranges of errors in Figs. 2 and 3 were calculated from the errors in the fluxes as in Vader \& Chaboyer (1994) using the formula:

$$
\delta I=\sqrt{N_{t o t}+\left(\delta n_{s k y} A\right)^{2}}
$$

where $N_{\text {tot }}$ is the flux from the galaxy in aperture $A$, and $\delta n_{s k y}$ is the standard deviation from the mean sky background determined in small apertures near the galaxy.

To calculate the stellar disk luminosity, we decomposed the images of the galaxies into components using the BUDDA code developed by de Souza et al. (2004) (version 2.2 which allows for the bulge, disk, bar, and central source components). In our analysis we evaluate the following components: the exponential disk $\mu_{d}(r)=\mu_{0}+1.086 r / r_{d}$, a bulge, and a bar with brightness distributed according to the Sersic law $\mu_{b}(r)=\mu_{e}+$ $c_{n}\left(\left(r / r_{e}\right)^{1 / n}-1\right)$. The decomposition results for the R-band profiles are shown in Figs. 4 and 5, respectively. The structural parameters determined for different bands are given in Table. 3. It contains the following data:

(1) galaxy name;

(2) the radial scale length of disk in arcsec;

(3) the central surface brightness of the disk in $m / \square^{\prime \prime}$;

(4) the effective radius of the bulge in arcsec;

(5) the effective surface brightness of the bulge in $m / \square^{\prime \prime}$;

(6) the Sersic index for the bulge;

(7) the effective radius of the bar in arcsec;

(8) the effective surface brightness of the bar in $m / \square^{\prime \prime}$;

(9) the Sersic index for the bar;

(10) the photometric band.

The images were not successfully decomposed for two galaxies: NGC 1569 and NGC 4214, both are without noticeable bulges, because their disk brightness profiles can be very poorly described by an exponential law. We show no data on these galaxies in Table 3). Nevertheless their observed profiles were considered when we photometrically so it remains close to the expected value for a given color. 

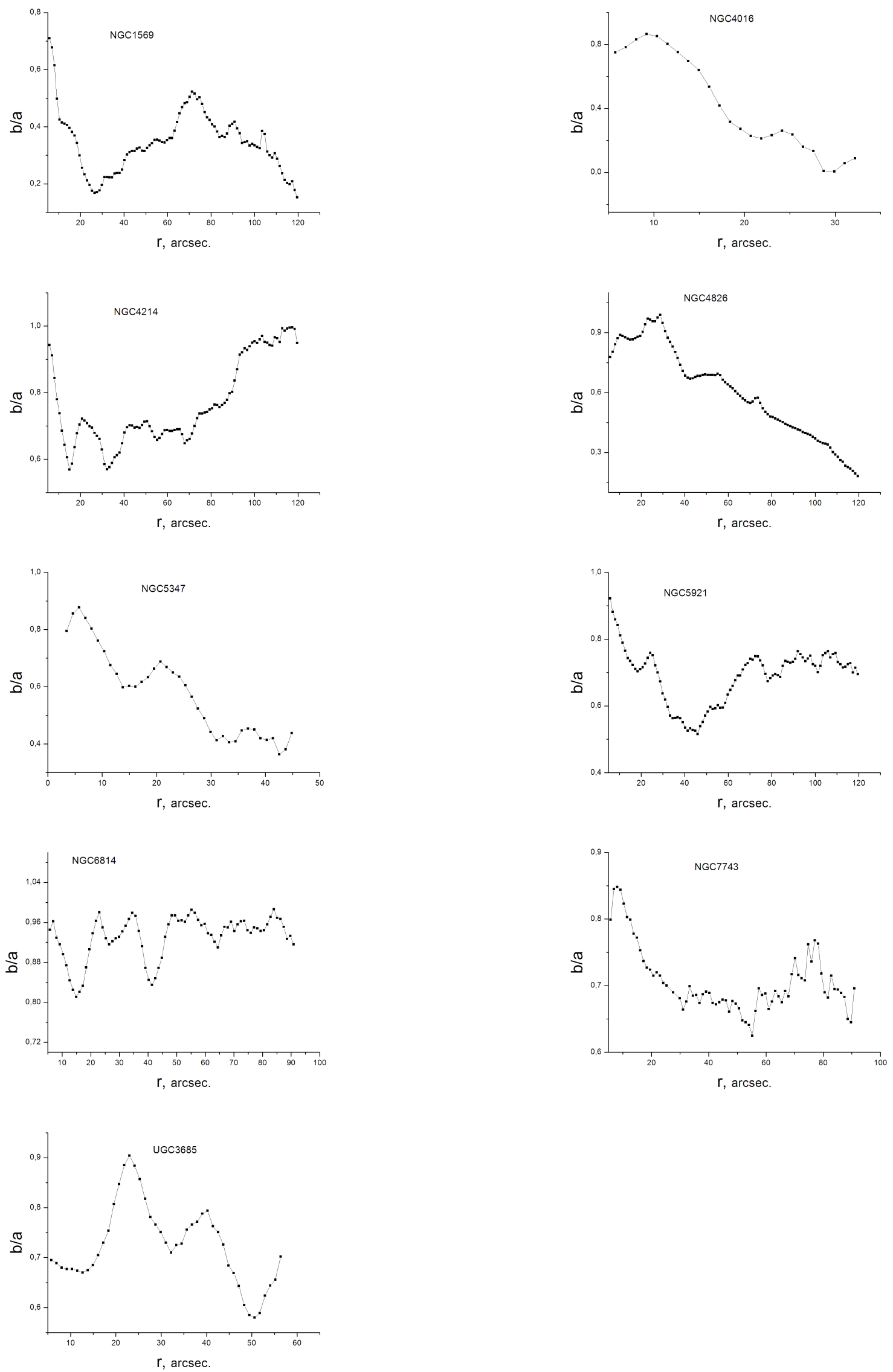

Fig. 1: Variations in the axial ratio of isophotes along the radius from $\mathrm{R}$-band images. 

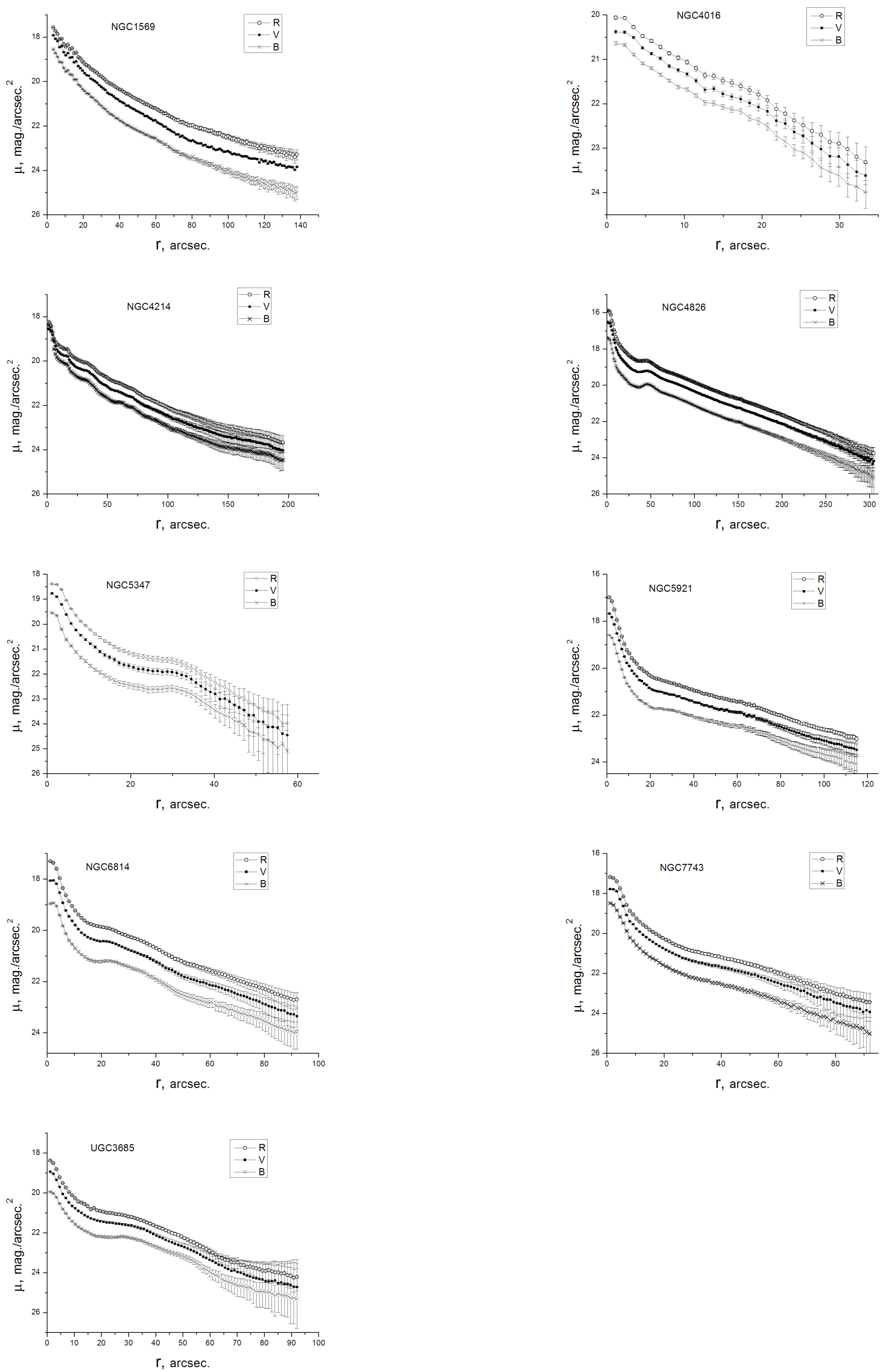

Fig. 2: Azimuthally averaged surface brightness profiles. 

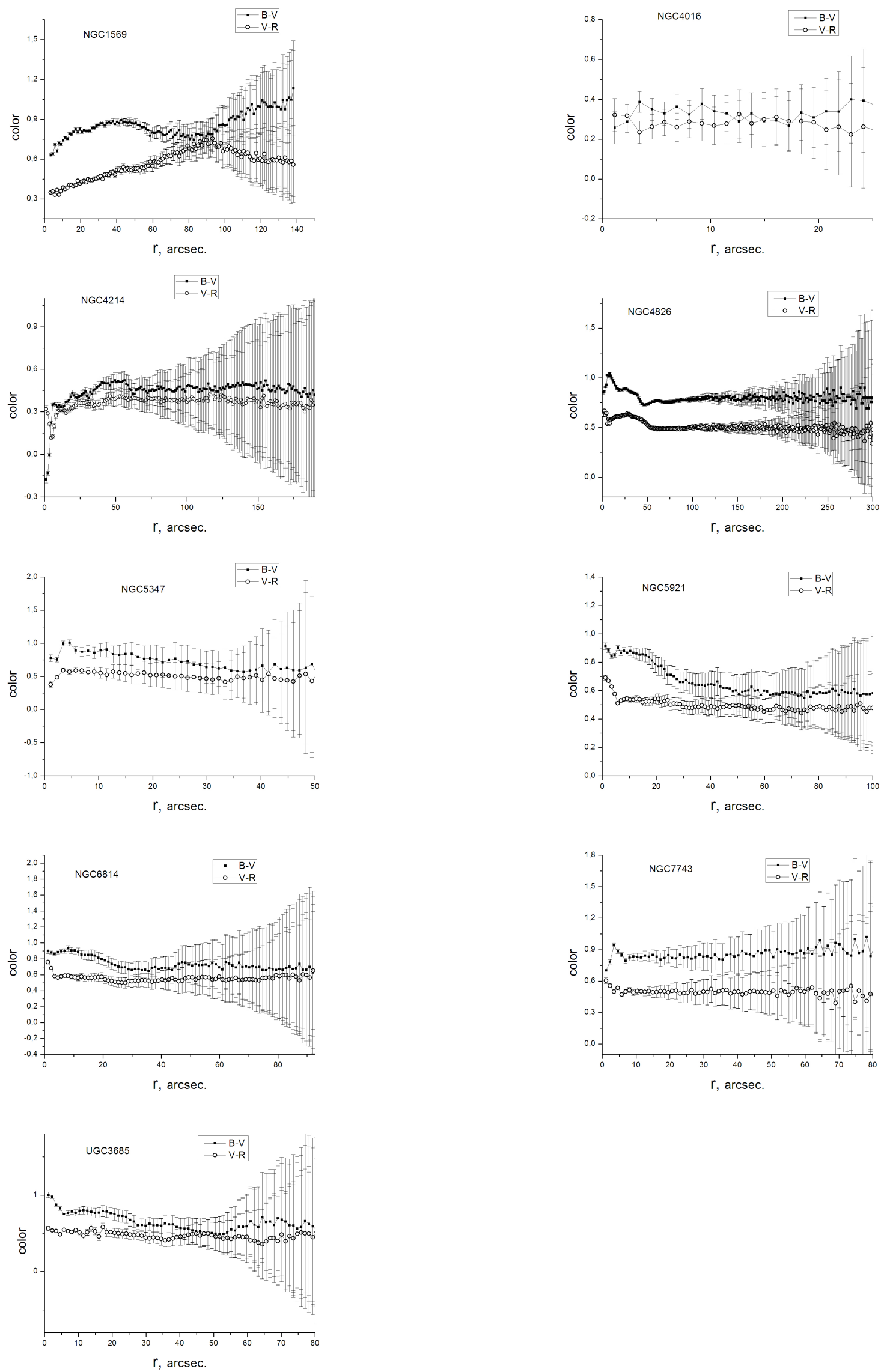

Fig. 3: Radial distributions of the azimuthally averaged colors. 

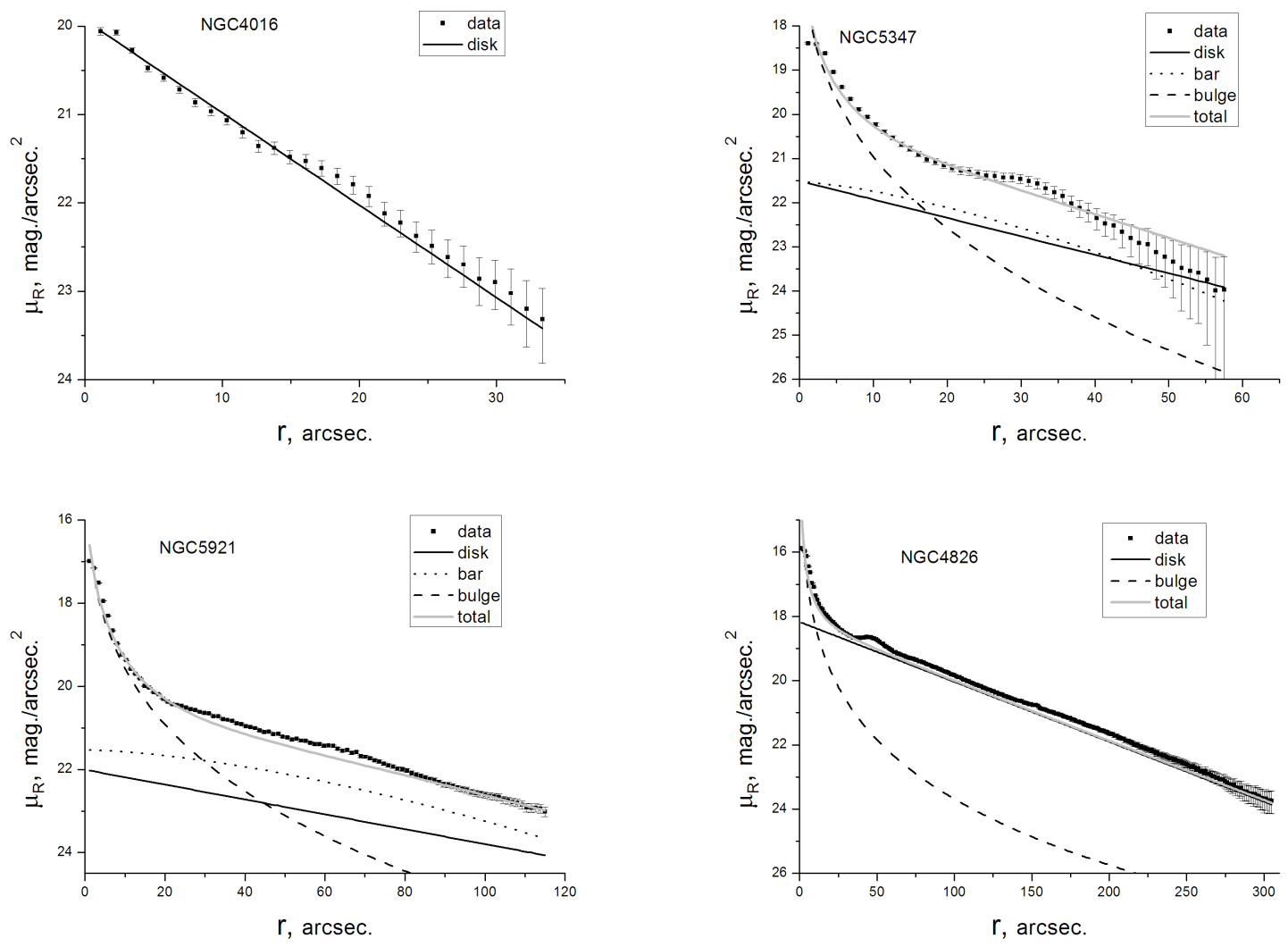

Fig. 4: The two-dimensional decomposition of the R-band surface brightness profiles into components (disk, bulge, and bar) using the BUDDA code. 

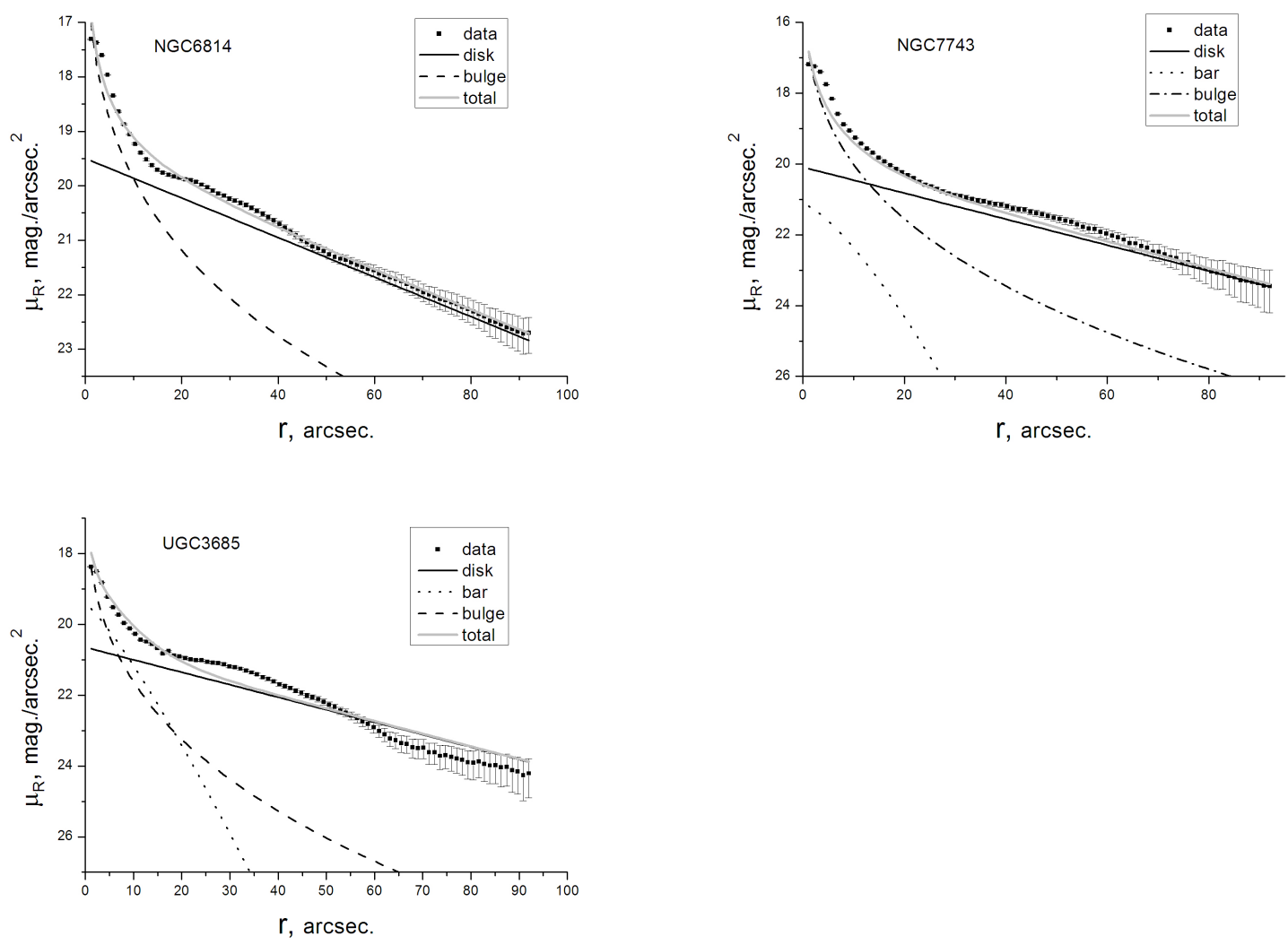

Fig. 5: Same as Fig. 4 for the galaxies UGC 3685, NGC 7743, and NGC 6814.

estimated the disk contribution to the total rotation curve.

The quality of the image decomposition is clearly demonstrated in Fig. 6 for NGC 5437. The image of this galaxy before and after the subtraction of the model image generated by the BUDDA code is shown (the model contains disk, bulge and bar). Both images have the same contrast. Only a faint ring and regions of enhanced brightness at the bar ends are noticeable in the residual image.

The BUDDA code reliably estimates the structural parameters of disks (see Figs. 4- 6, Table 4), although, according to Gadotti (2008), the bulge-to-disk luminosity ratio can be systematically overestimated by $5 \%$ while the relative disk luminosity can be systematically underestimated. Below we also consider the total $M / L$ ratios independent of the photometric decomposition results, along with the mass-to-light ratios for the disks. 


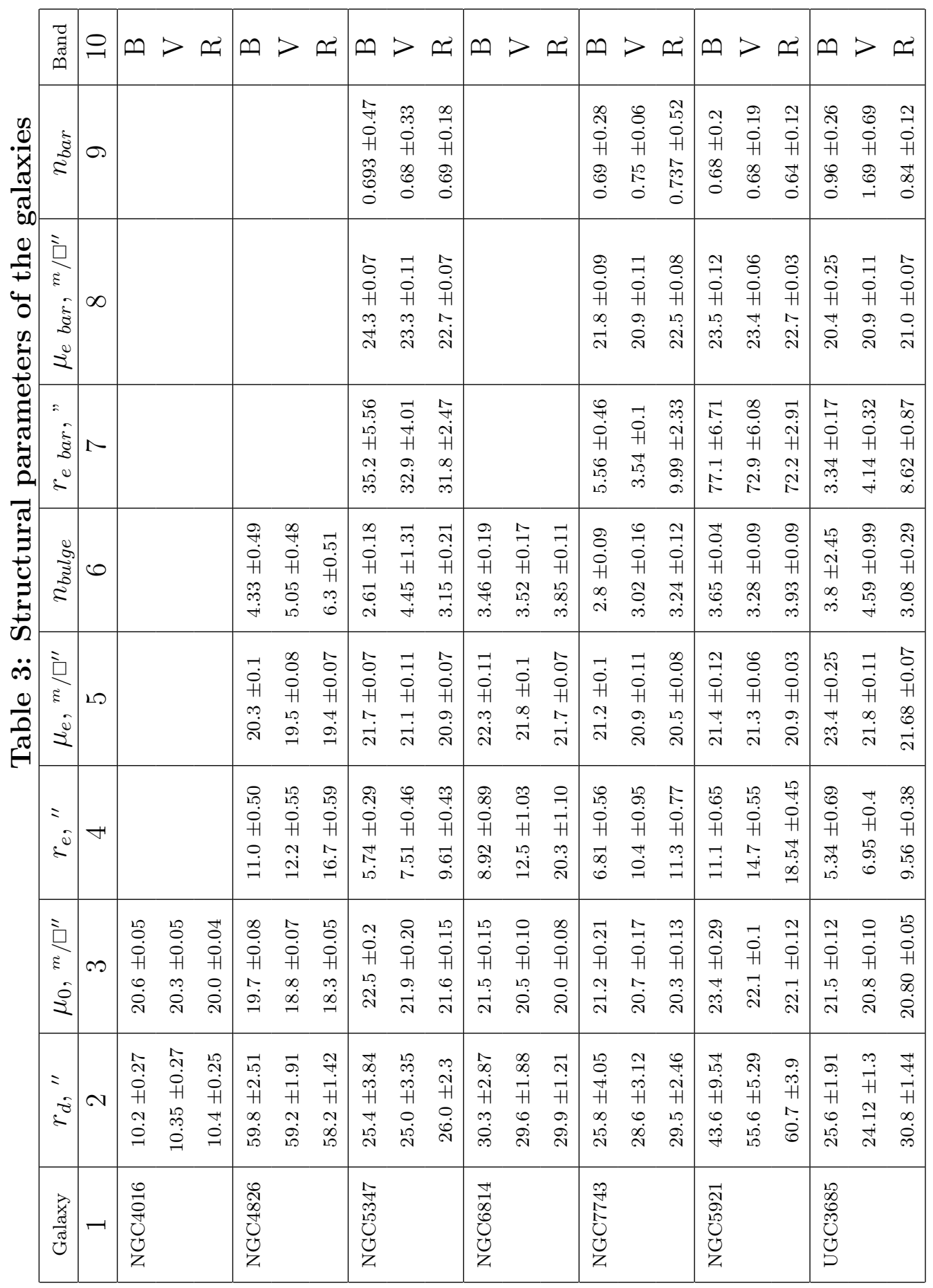



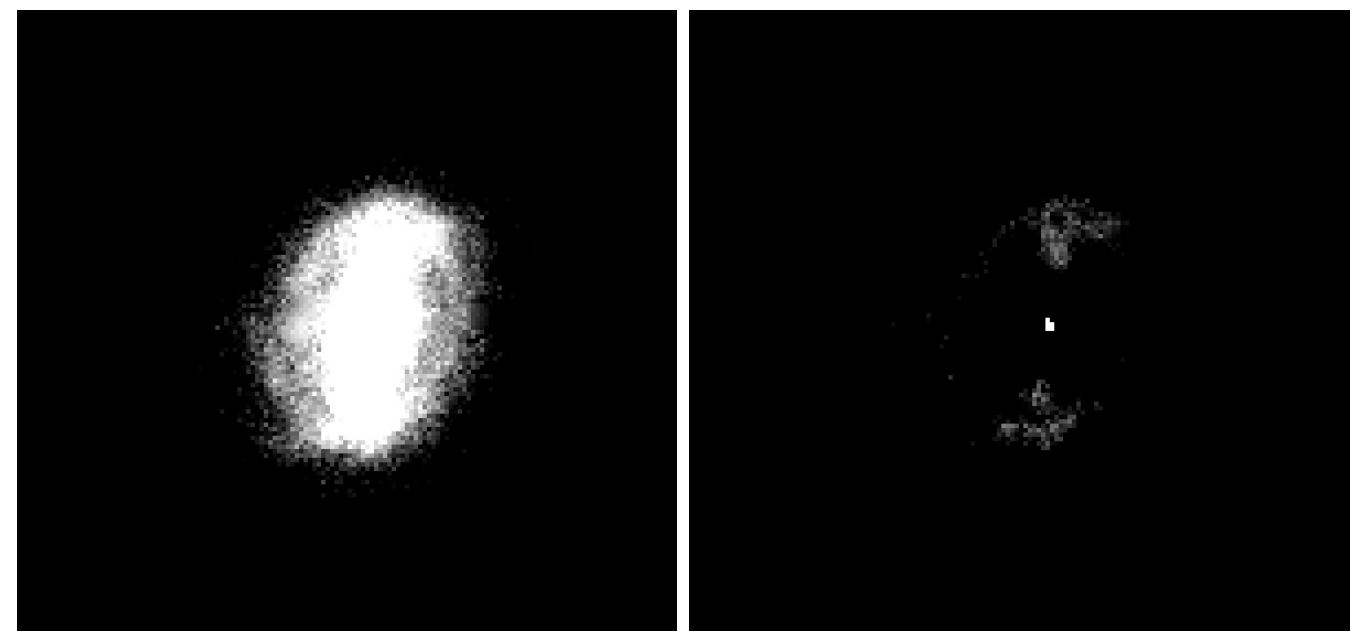

Fig. 6: The R-band image of the galaxy with a bright bar NGC 5347 before and after the subtraction of the model image generated by the BUDDA. Both images have the same contrast.

\section{COMPARISON WITH OTHER PUBLICATIONS}

We compare the surface brightness profiles with results by other authors. In Fig. 7, the profiles of all galaxies (except NGC 1569) were corrected for extinction in the Galaxy, but were not corrected for the disk inclination. Fig. 7 shows good agreement between our estimates and published results. An exception is NGC 1569, which exhibits a significant discrepancy at the periphery between our R-band brightness profile and those from Swaters \& Balcells (2002) and Stil \& Israel (2002). This discrepancy may be explained by diferent procedures we used for subtraction of numerous foreground stars for this galaxy. There is also a noticeable discrepancy at the periphery between our R-band profile of NGC 6814 and that from de Robertis et al. (1998). However, there is a good agreement with the profile from Sánchez-Portal et al. (2000) observed in the same band (see Fig. 7). In the remaining cases, the difference between our profiles and those of other authors does not exceed $0.3^{m}$. This is comparable to the typical errors in the surface brightness estimates far from the center.

\section{COMPARISON OF THE PHOTOMETRIC AND DYNAMICAL DISK MASS ESTIMATES}

We choose two conditions that allow us to suspect the existence of a discrepancy between the photometric and dynamic mass estimates of the stellar population of a galaxy: (i) The photometric $M / L$ of stellar population of a galaxy within some radius (taken within 

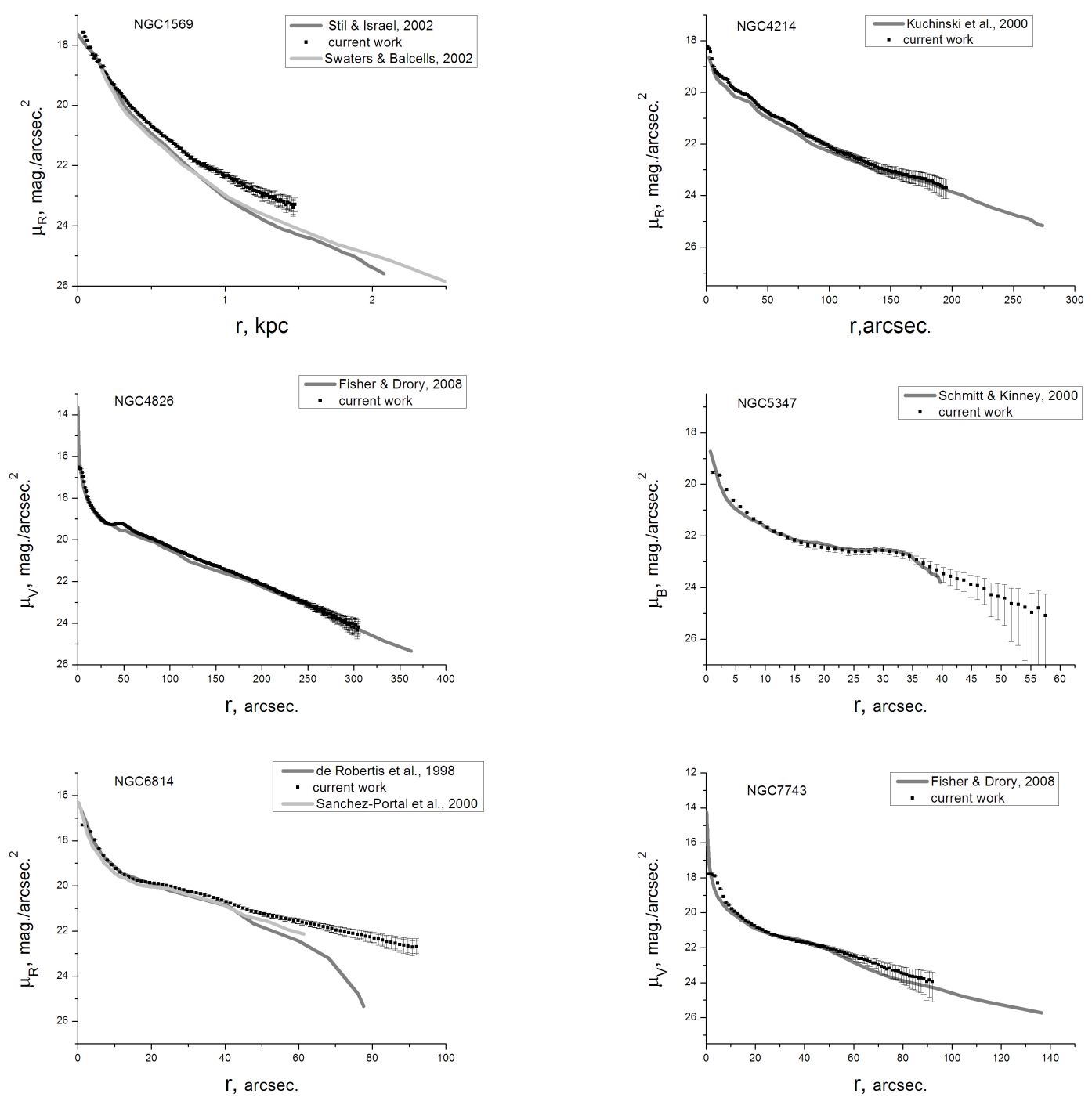

Fig. 7: Comparison of the $B, V, R$ brightness profiles with the results of other authors. 
four radial scale lengths $r_{d}$ ) obtained from the total color index, exceeds the dynamical estimate of $M / L$ determined from the relation $(M / L)_{d y n}=4 v_{m a x}^{2} r_{d} /\left(G L_{t o t}\right)$, where $L_{t o t}$ is the total luminosity (including bulge) and $v_{\max }$ is the maximum rotational velocity of the galaxy.

(ii) The maximum rotational velocity of the disk component $v_{\max }$ disk estimated from the photometric disk mass and scale length exceeds the observed maximum rotational velocity of the galaxy $v_{\max }$. The value of $v_{\max }$ disk can be determined from the radial surface density profile $\sigma(R)$ obtained from the brightness distribution and the model $M / L$ ratio estimated from the measured color index. The rotation curve in spiral galaxies is usually a constant beyond $r=(1.5-2) r_{d}$. Hence we assume that $v_{\max }$ is close to the rotation velocity of galaxy at $r \approx 2 r_{d}$, where the disk component of rotation curve approaches $v_{\max }$ disk.

For all galaxies except NGC 4214 and NGC 1569 the disk $M / L$ ratio was assumed to be constant along the radius, while the radial change of $M / L$ in the two mentioned objects was determined from the color profiles. For an exponential disk density distribution adopted for all galaxies except NGC 4214 and NGC 1569, $v_{\text {max disk }} \approx 0.623\left(G M_{d} / r_{d}\right)^{0.5}$, where $M_{d}=2 \pi G \sigma_{0} r_{d}^{2}$ is the disk mass and $\sigma_{0}$ is the central surface density of the disk.

Galaxies with abnormally low $M / L$, which is at least a factor of 3 lower than the expected ratio for a given $(B-V)_{0}$ color, are rare - they account for about $3 \%$ of the number of galaxies of S0 and later types which have the measured rotational velocities (from the HI line width) and $(B-V)_{0}$ colors (see Fig. 1 in Saburova et al. 2009). In fact, their number may be considerably smaller. There are two factors which may be responsible for the discrepancy between the dynamical and photometric disk mass estimates:

(i) errors in the estimates of observed quantities, such as the circular velocity, the inclination, the distance to the galaxy, the luminosity and color;

(ii) inconsistency of the galaxy's stellar population with the evolutionary population synthesis model used for the $M / L$ estimate: the ignorance of starburst, low metallicity Z of the stellar population, and/or an anomalous stellar IMF. Below we consider each of these factors in our galaxies.

Note that all galaxies of our sample except NGC 1569 and NGC 4016 follow the 
sequence of $(B-V)-(V-R)$ colors for normal galaxies derived by Buta \& Williams (1995). This suggests the absence of significant peculiarities in the star formation history and confirms the applicability of population synthesis models (Bell and de Jong 2001). The star formation in NGC 1569 is very active, then we apply the $M / L$ color relation including a starburst. The second galaxy, NGC 4016, was included in the sample due to the unrealistically low $(B-V)_{0}$ given by NED, but it is not confirmed in our work, so we conclude that this is not a galaxy with an abnormally low $M / L$ (see below).

NGC1569 is a Magellanic-type irregular dwarf starburst galaxy. Active star formation in this object is probably associated with tidal interaction (Grocholski et al. 2008). According to Recchi et al. (2006), the chemical abundance in this galaxy is consistent with the assumption that there were long-lasting episodes of quiet star formation completed by series of starbursts. Since NGC 1569 lies near the Galactic plane, the extinction correction is very uncertain for it: the correction coefficients in Schlegel et al. (1998) and Burstein and Heiles (1982) for the $B$ band differ by $1^{m}$. This galaxy is a probable cause of the low mass-to-light ratio estimate. Therefore, we can adopt the photometric $K s$-band profile from Vaduvescu et al. (2006), along with the $B, V, R$ magnitudes, in order to estimate the disk mass from the photometry and its contribution to the rotation curve. Using the $K s$ band also allows us to reduce the effects of intense star formation on the model estimate of the mass-to-light ratio.

The ionized gas velocity field of NGC 1569 obtained within the framework of the GHASP project (Epinat et al. 2008) covers the central parts of the galaxy and shows no evidence of rotation. However, the rotation curve determined by Stil and Israel (2002) in the H I line allows the maximum rotational velocity $v_{\max }$ to be estimated. We compared $v_{\max }$ with the maximum rotational velocity of the disk component of the rotation curve $v_{\text {max disk }}$ by estimating the disk mass from photometric data. The disk density profile needed for this purpose was calculated from the $K s$ and $R$ brightness profiles as well as from the $(V-R)$ color distribution and the total $(J-K)$ color obtained by Vaduvescu et al. (2006). We determined the expected $M / L_{R}$ and $M / L_{K}$ ratios for the disk (see Table 4) from the color indices following the models of Bell and de Jong (2001) calculated for a modified Salpeter IMF with the inclusion of a starburst 5 Taking into account the

\footnotetext{
${ }^{5}$ We took the starburst duration $0.5 \mathrm{Gyr}$ and the fraction of young stars is $10 \%$ of the total mass of
} 
radial brightness profiles in these photometric bands, we obtained the radial disk surface density profile and the corresponding values of $v_{\max }$ disk (see Table 4). It follows that $v_{\text {max disk }}$ determined from $K s$ photometry turns out to be close to the observed maximum rotational velocity. Hence we may conclude that this object does not have an abnormally low $M / L$. Note that the mass of its dark halo within the optical boundaries should be small compared to the disk mass, as suggested by the closeness of the $v_{\max }$ disk and $v_{\max }$ estimates.

NGC4016 is an SBd-type galaxy. We included it in our sample due to its unrealistically low color index $(B-V)=0.03$ in the NED, which we do not confirm (see Table 2). The rotational velocity $v_{\max \text { disk }}$ determined from the photometric density estimate using our color and luminosity measurements turns out to be lower than the total rotational velocity $v_{\max }$ (see Table 4). This gives no grounds for attributing it to objects with anomalous $M / L$.

NGC4214, just as NGC 1569, is a Magellanic type irregular galaxy with a bar. The main problem related to the mass estimation for this galaxy is an unreliably determined inclination (see Fig. 2). The axial ratio of NGC 4214 changes along the radius from 0.7 to 0.94 , which corresponds to the inclinations $i_{1}=45^{\circ}$ and $i_{2}=20^{\circ}$. The rotational velocity $v_{\max }$ disk found from the R-band brightness profile and the $(B-R)$ and $(B-V)$ colors for a modified Salpeter IMF, including a starburst similar to that adopted for NGC 1569, is compared in Table 4 with the observed rotational velocity $v_{\max }$ of the galaxy for both inclination angles. As follows from our estimates, $v_{\max }$ may be either higher or lower than $v_{\max \text { disk }}$ if to choose $i_{2}=20^{\circ}$ or $i_{1}=45^{\circ}$, respectively. Obviously, the observed flattening of the galaxy's inner part is distorted by the presence of a bar, so the peripheral values of $b / a$ are preferable for the inclination estimate. However, the contradiction between the photometric and dynamical models is retained even at $i_{2}=20^{\circ}$ : in this case, in spite of $v_{\max }>v_{\max }$ disk, the radius at which the disk component of the rotation curve, obtained from photometry data, has a maximum $(R \approx 2 \mathrm{kpc})$ turns out to be a factor of 3 smaller than the radius where the observed rotation curve derived by Allsopp (1979) reaches its maximum. Thus, the disk with the photometrically calculated density distribution is inconsistent with the the stellar population. 
observed rotation curve. This conclusion remains valid even if we take into account the low linear resolution of the H I data in Allsopp (1979) $(\Delta R \approx 2 \mathrm{kpc})$. However, there remains the possibility that the discrepancy between $v_{\max }$ disk and the rotation velocity in the central part of the galaxy can be associated with noncircular motions of gas due to the presence of a bar. Therefore, although an anomalous stellar composition in NGC 4214 remains quite possible, the available data do not allow a reliable $M / L$ estimate to be obtained for this galaxy. A more detailed study of its velocity field is required.

NGC4826 is an Sab-type galaxy with a thick dust lane northeast of the nucleus. The rotation of the outer gas disk in NGC 4826 is opposite to that of the inner one (Braun et al. 1994). A detailed study of the $H_{\alpha}$ kinematics shows that the transition between the two disks occurs near the dust lane at a distance $50^{\prime \prime}<r<70^{\prime \prime}$ from the center (Rubin 1994). The velocity $v_{\max }$ disk calculated from the R-band photometric parameters of the disk (see Table 3$)$ and the $(B-V)$ and $(B-R)$ colors beyond the dust lane turns out to be higher than the maximum rotational velocity $v_{\max }$ (see Table 4). This conclusion remains valid even if we use the model by Bell and de Jong (2001) with lower metallicity $(Z=0.008)$. Thus, our photometric data agree with the abnormally low $M / L$ ratio in this galaxy.

NGC 5347 is an Sab-type galaxy with a bar. The $P V$ - diagram constructed from optical observations has a large scatter of points (Marquez et al. 2004) and, therefore, does not allow $v_{\max }$ to be determined reliably. However, the galaxy's rotational velocity estimated from the $\mathrm{H}$ I line width $\left(W_{20}\right.$ from the RC3 catalog by de Vaucouleurs et al. 1991) turns out to be lower than that for the disk component of the rotation curve determined from the radial $R$-band surface brightness profile (after the subtraction of the bulge contribution) using the color indices of the galaxy's outer regions (see Table 4 . Thus, an abnormally low mass-to-light ratio for the disk can actually take place in this case. This object requires a careful study and, first of all, it is necessary to obtain a more extended rotation curve.

NGC 5921 is an Sbc-type galaxy with a noticeable bar surrounded by a ring. As for NGC 4214, the inclination estimates for this galaxy are contradictory. According 
to Hernandez et al. (2005), the isophotes ellipticity leads to $i=36.5^{\circ}$. Our photometric data give $i=43^{\circ}$ (see Fig. 2), which agrees with the inclination determined kinematically from $H_{\alpha}$ observations (Hernandez et al. 2005). In Table 4, the rotational velocity $v_{\text {max disk }}$ determined photometrically in the same way as for NGC 5347 (see above) is compared with the two values of rotation velocity $v_{\max }$ found from $H_{\alpha}$ measurements for $i=43^{\circ}$ and $i=36.5^{\circ}$ for radial distance corresponding to $v_{\max \text { disk. }}$. As we see from Table 4, for $i=36.5^{\circ} v_{\max \text { disk }}$ is lower than $v_{\max }$, while the opposite conclusion is true for $i=43^{\circ}$. Since our inclination estimates agree with the latter value, an abnormally low mass-to-light ratio for the disk of NGC 5921 remains quite possible. However, an underestimation of the circular rotational velocity caused by the bar cannot be ruled out. This is suggested by the fact that the rotational velocity estimated from the H I line width (from the RC3 catalog) for $i=43^{\circ}$ is $v_{H I}=136 \mathrm{kms}^{-1}$, which exceeds $v_{\text {max disk }}$ (see Table 4). Thus, the abnormally low $M / L$ for NGC 5921 is most likely related to noncircular motions and not to stellar composition anomalies.

NGC 6814 is an SABb-type galaxy with a ring. The inclination of NGC 6814 is low (the photometric axial ratio gives $i=17^{\circ}$ ). However, $i=8^{\circ}$ is required for $v_{\max }$ determined from the rotation curve in the $\mathrm{HI}$ line to become approximately equal to $v_{\max }$ disk. Such a low inclination does not correspond to the photometric estimates (see Fig. 2). The presence of a bar hardly may change the result, because its presence is evident only in the central region $(R<1 \mathrm{kpc})$, while the disk component of the rotation curve has a maximum at $R \approx 3 \mathrm{kpc}$. Therefore, the conclusion about an abnormally low $M / L$ of this galaxy remains preferable.

NGC 7743 is an S0-a galaxy with a bar. As in the previous two cases, the abnormally low $M / L$ for the disk may be caused by the influence of the bar on the rotation velocity. It may also partially be attributed to the galaxy's low metallicity $\left(Z=0.008\right.$ according to Katkov et al. 2011). In Table 4 , the two values of $v_{\max }$ disk obtained photometrically for two different metallicities are compared with $v_{\max }$ reached at $R=5 \mathrm{kpc}$. For $Z=0.008$, the velocities $v_{\max }$ disk and $v_{\max }$ turn out to be close (see Table 4). Consequently, as has been noted above, the abnormally low $M / L$ in NGC 7743 is not evident if to take into account a low metallicity of the stellar population and the influence of the bar on the $v_{\max }$ estimate. 
UGC 03685 is an Sb-type galaxy. Just as NGC 6814 and NGC 5921, it has a bar and a ring, while having a small inclination. The inclination determined from the $H_{\alpha}$ kinematics is very uncertain $\left(i=12^{\circ} \pm 16\right)$ (Epinat et al. 2008). According to our photometric data, $i=40^{\circ} \pm 15$ (see Fig. 2), which is consistent with the NED $\left(33^{\circ}\right)$ and Hyperleda $\left(55^{\circ}\right)$ estimates. In Table 4 , the disk rotational velocity $v_{\text {max disk }}$ estimated photometrically is compared with the total rotational velocities $v_{\max }$ corresponding to $i_{1}=12^{\circ}$ and $i_{2}=40^{\circ}$. The conclusion about an abnormally low mass-to-light ratio for the disk of UGC 03685 is confirmed if we take the photometrically estimated inclination $i_{2}$ and is not confirmed for $i_{1}$. If we take into account the large error in the kinematic inclination estimate, then the conclusion about an abnormally low $M / L$ remains possible, though uncertain.

Table 4 contains the following data:

(1) galaxy name;

(2) maximum rotational velocity of the disk-related component of the rotation curve $v_{\text {max disk }}$ determined photometrically based on the Bell, de Jong (2001) models;

(3) maximum rotational velocity $v_{\max }$ derived from direct observations (for NGC 7743, NGC 5921, NGC 6814, and UGC 3685, this is the velocity of rotation at the distance $R=2 r_{d}$, where the disk contribution to the rotation curve is maximal);

(4) reference to the source of the rotation curve;

(5) note;

(6) photometrically determined mass-to-light ratio for the stellar population of the disk (in the R- band) from the model by Bell, de Jong (2001);

(7) total dynamical mass-to-light ratio for the galaxy in the R-band within the optical radius $R=4 r_{d}$ (for NGC 1569 and NGC 4214 it is within $R_{25}$ ); for NGC 1569 the second row of the table gives the total $M / L_{K s}$ ratio;

(8) total mass-to-light ratio for the galaxy $M / L_{R}$ calculated from the models by Bell, de Jong (2001) and the total $(B-R)_{0}$ color (for NGC 1569, the second row gives $M / L_{K s}$ calculated from the total $(J-K s)_{0}$ color $)$.

\section{CONCLUSIONS}


Table 4: Comparison of $v_{\max }$ disk with $v_{\max }$ and $M / L$ estimates

\begin{tabular}{|c|l|l|c|c|c|c|l|}
\hline Galaxy & $\begin{array}{l}v_{\text {max disk }}, \\
\mathrm{km} \mathrm{s}^{-1}\end{array}$ & $\begin{array}{l}v_{\text {max }}, \\
\mathrm{km} \mathrm{s}^{-1}\end{array}$ & Ref. & Note & $\left(M / L_{R}\right)_{d}$ & $M / L_{R \text { dyn }}$ & $M / L_{R \text { mod }}$ \\
$(1)$ & $(2)$ & $(3)$ & $(4)$ & $(5)$ & $(6)$ & $(7)$ & $(8)$ \\
\hline NGC1569 & 60 & 43 & {$[31]$} & $\mathrm{R}$ & 0.16 & 0.146 & 0.26 \\
NGC1569 & 45 & 43 & {$[31]$} & $\mathrm{Ks}$ & 0.49 & 0.8 & 0.49 \\
\hline NGC4016 & 73 & 78 & {$[8]$} & - & 0.8 & 1.53 & 0.86 \\
\hline NGC4214 & 75 & 42 & {$[38]$} & $i=40^{\circ}$ & 0.8 & 0.3 & 0.73 \\
NGC4214 & 75 & 79 & {$[38]$} & $i=20^{\circ}$ & 0.8 & 1.06 & 0.73 \\
\hline NGC4826 & 256 & 154 & {$[4]$} & $Z=0.02$ & 2.25 & 1.06 & 2.24 \\
NGC4826 & 241 & 154 & {$[4]$} & $Z=0.008$ & 2 & 1.06 & 2.02 \\
\hline NGC5347 & 111 & 56 & {$[40],[24]$} & $Z=0.02$ & 1.5 & 0.65 & 2.00 \\
NGC5347 & 103 & 56 & {$[40],[24]$} & $Z=0.008$ & 1.3 & 0.65 & 1.79 \\
\hline NGC5921 & 139 & 111 & {$[33]$} & $i=43^{\circ}$ & 1.5 & 1.75 & 1.77 \\
NGC5921 & 139 & 127 & {$[33]$} & $i=36.5^{\circ}$ & 1.5 & 2.3 & 1.77 \\
\hline NGC6814 & 148 & 120 & {$[23]$} & - & 1.75 & 1.07 & 2.17 \\
\hline NGC7743 & 135 & 118 & {$[17]$} & $Z=0.02$ & 2.28 & 2.3 & 2.37 \\
NGC7743 & 125 & 118 & {$[17]$} & $Z=0.008$ & 2 & 2.3 & 2.15 \\
\hline UGC03685 & 86 & 101 & {$[20],[39]$} & $i=12^{\circ}$ & 1.25 & 3.37 & 1.69 \\
UGC03685 & 86 & 33 & {$[20],[39]$} & $i=40^{\circ}$ & 1.25 & 0.35 & 1.69 \\
\hline
\end{tabular}

We present the results of our surface photometry in the $\mathrm{B}, \mathrm{V}$ and $\mathrm{R}$ bands for nine disk galaxies in which the discrepancy between low M/L ratio and color of stellar population is suspected. We obtain the photometric profiles, the radial profiles of the color indices, the position angle, and the flattening of isophotes. We decomposed the images using the estimation of photometric parameters for individual components (bulge, bar and disk) of the galaxies. We apply two methods for the dynamical and photometric disk mass estimates: by checking whether the colors agree with the total $M / L$ ratios of the galaxies, which may be considered as an upper limit of $M / L$ for the stellar population, and by comparing the observed rotational velocities of the galaxies with the maximum circular velocity of the disk expected from its photometric parameters. Our results show that there are no obvious contradictions between the dynamical and photometric mass estimates in most considered objects because the abnormally low dynamical $M / L$ estimates can be naturally explained by the uncertainty in determining the rotational velocity or by the errors in photometry. At the same time our photometric data for NGC 4826, NGC 6814, NGC 5347, and with lesser confidence for UGC 03685 and NGC 4214 suggest that these galaxies may actually be too "light" for their luminosity, and hence may have the IMF defficient by low-mass stars. The presence of dark matter in the galaxies which was ignored 
in our study would only increase the discrepancy between the dynamical and photometric $M / L$ estimates. The galaxies we discuss require a more complete study: first of all, more careful measurements of the rotational velocity far from the center are needed.

The low values of the mass-to-light ratio in galaxies, even if this is not a result of anomalous stellar population, suggest the low dark-to-luminous matter mass fraction in them.

\section{ACKNOWLEDGMENTS}

We wish to thank R. Swaters who kindly provided the brightness profile of NGC 1569. We also wish to thank R.E. de Souza and D.A. Gadotti for the opportunity to use the BUDDA code. We are grateful to Hyperleda support team for the opportunity to use this database. Based on observations obtained with the Apache Point Observatory 0.5meter telescope, which is owned and operated by the Astrophysical Research Consortium. This work was supported by Russian Foundation for Basic Research, grant 11-02-12247.

\section{References}

[1] N. Bastian, K.R. Covey, M.R. Meyer, astro-ph: 1001.2965v2 (2010)

[2] E. F. Bell , R. S. de Jong, Astrophys. J., 550, 212 (2001)

[3] D. Bizyaev, S. Mitronova, Astrophys. J. 702, 1567 (2009)

[4] R. Braun, R.A.M. Walterbos, R.C.J. Kennicutt, L.J. Tacconi, Astrophys. J., 420, $558(1994)$

[5] D. Burstein \& C. Heiles, Astron.J., 87, 1165 (1982)

[6] R. Buta, K.L. Williams, Astron. J., 109, 543 (1995)

[7] O. Vaduvescu, M. G. Richer, M. L. McCall, Astron. J., 131, 1318 (2006)

[8] G.A. van Moorsel, Astron. Astrophys. S, 54, 19 (1983)

[9] J. P. Vader, B. Chaboyer, Astron. J., 108, 1209 (1994)

[10] D.A. Gadotti, MNRAS, 384, 420 (2008) 
[11] G. Gilmore, Starburst Galaxies: Near and Far, Proceedings of a Workshop held at Ringberg Castle, Edited by L. Tacconi and D. Lutz. Heidelberg: Springer-Verlag, 2001., p.34

[12] A. J. Grocholski, A. Aloisi, R. P. van der Marel et al., Astrophys. J., 686L, 79 (2008)

[13] J. Dabringhausen, M. Fellhauer, P. Kroupa, astro-ph: 0912.2998v2 (2010)

[14] M. M. de Robertis, K. Hayhoe, H. K. C. Yee, Astrophys. J. Suppl. Ser., 115,163 (1998)

[15] R. E. de Souza, D. A. Gadotti, S. dos Anjos, Astrophys. J.Suppl. Ser., 153, 411 (2004)

[16] A. V. Zasov, A. V. Khoperskov, A. S. Saburova, Astronomy Letters 37, 374 (2011)

[17] I. Katkov, A. Moiseev, O. Sil'Chenko, Astrophys. J. 740, 83 (2011)

[18] P. Kroupa, Science, 295, 82 (2002)

[19] L.E. Kuchinski, W.L. Freedman, B.F. Madore et al., Astrophys. J.Suppl. Ser., 131, $441(2000)$

[20] D. A. Kornreich, M. P. Haynes, R. V. E. Lovelace, L. van Zee, Astron. J., 120, 139 (2000)

[21] A.U. Landolt, Astron. J., 137, 4186 (2009)

[22] A.U. Landolt, Astron. J., 104, 340 (1992)

[23] H.S. Liszt, J.M. Dickey, Astron. J., 110, 998 (1995)

[24] I. Marquez, F. Durret, J. Masegosa et al., Astron. Astrophys., 416, 475 (2004)

[25] G.R. Meurer O.I. Wong, J.H. Kim et al., Astrophys. J., 695, 765 (2009)

[26] S. Recchi, G. Hensler, L. Angeretti, F. Matteucci, Astron. Astrophys., 445, 875 (2006)

[27] V. C. Rubin, Astron. J., 107, 173 (1994)

[28] A. S. Saburova, E. S. Shaldenkova, A. V. Zasov, Astronomy reports, 53, 801 (2009)

[29] M. Sánchez-Portal, A.I. Díaz, R. Terlevich et al., MNRAS, 312, 2S (2000)

[30] R. A. Swaters \& M. Balcells, Astron. Astrophys., 390, 863 (2002) 
[31] J. M. Stil, F. P. Israel, Astron. Astrophys., 392, 473 (2002)

[32] D.B. Fisher, N. Drory, Astron. J., 136, 773 (2008)

[33] O. Hernandez, C. Carignan1, P. Amram, L. Chemin and O. Daigle, MNRAS, 360, $1201(2005)$

[34] E.A. Hoversten, K. Glazebrook, Astrophys. J., 675, 163 (2008)

[35] Khoperskov A., Bizyaev D., Tiurina N., Butenko, M. Astron. Nachr. 331, 731 (2010)

[36] D. J. Schlegel, D. P. Finkbeiner, M. Davis, Astrophys. J., 500, 525 (1998)

[37] H. R. Schmitt, A. L. Kinney, Astrophys. J.Suppl. Ser., 128, 479 (2000)

[38] N. J. Allsopp, MNRAS, 188, 765 (1979)

[39] B. Epinat, P. Amram, M. Marcelin et al., MNRAS, 388, 500 (2008)

[40] THIRD REFERENCE CATALOGUE OF BRIGHT GALAXIES, G. de Vaucouleurs, A. de Vaucouleurs, H. G. Corwin, Volume 1-3, XII, 2069 pp., Springer-Verlag Berlin Heidelberg New York (1991)

[41] Hyperleda: http://leda.univ-lyon1.fr/

[42] Ned: http://www.ned.ipac.caltech.edu/ 